\title{
Phenotypic methods for screening carbapenem-resistant Enterobacteriaceae and assessment of their antimicrobial susceptibility profile
}

\author{
Danielly da Costa Silva ${ }^{[1],[2],}$ Roberta Filipini Rampelotto ${ }^{[1],[2], V i n i ́ c i u s ~ V i c t o r ~ L o r e n z o n i ~}{ }^{[1],}$ \\ Silvana Oliveira dos Santos ${ }^{[1],[2], J u l i a n a ~ D a m e{ }^{[1],[2],}}$ \\ Manfredo Hörner ${ }^{[3]}$ and Rosmari Hörner ${ }^{[1]}$
}

\begin{abstract}
[1]. Departamento de Análises Clínicas, Universidade Federal de Santa Maria, Santa Maria, RS, Brasil. [2]. Programa de Pós-Graduação em Ciências Farmacêuticas, Universidade Federal de Santa Maria, Santa Maria, RS, Brasil. [3]. Departamento de Química, Universidade Federal de Santa Maria, RS, Santa Maria, Brasil.
\end{abstract}

\begin{abstract}
Introduction: In this study, we used phenotypic methods to screen carbapenem-resistant Enterobacteriaceae (CREs) and evaluated their antimicrobial sensitivity profile. Methods: One hundred and seventy-eight CREs were isolated at a university hospital in south Brazil in a one-year period. Samples were assessed using disk diffusion tests with inhibitors of $\beta$-lactamases such as phenylboronic acid (AFB), cloxacillin (CLOXA), and ethylenediaminetetraacetic acid (EDTA). Strains with differences in zone diameters $\geq 5 \mathrm{~mm}$ for disks supplemented or not were considered producers of carbapenemases. Results: Klebsiella pneumoniae was the most prevalent CRE, which appeared in $80.3 \%$ cases $(\mathrm{n}=143)$. Among clinical materials, the rectal swab was responsible for $43.4 \%$ of the isolations $(n=62)$, followed by urine $(18.9 \% ; n=27)$. Among the CREs identified in this study, the growth of $56.7 \%(\mathrm{n}=101)$ isolates, which were putative producers of Klebsiella pneumoniae carbapenemase (KPC), were inhibited by AFB, whereas 7.3\% $(\mathrm{n}=13)$ isolates were inhibited by both AFB and CLOXA and were considered as putative producers of plasmid-mediated AmpC; approximately 3.4\% $(n=6)$ were inhibited by EDTA, which possibly produced metallo- $\beta$ lactamase. Lastly, 32.6\% $(n=58)$ cases showed negative results for AFB, CLOXA, and EDTA sensitivity, and represented another class of $\beta$-lactamases and/or mechanism of resistance. Conclusions: Phenotypic screening of CREs is important for clinical laboratories that monitor outbreaks of resistant microbes. Phenotypic tests that use carbapenemase inhibitors and enhancers such as AFB, CLOXA, and EDTA are necessary since they are good screening methods for the detection of carbapenemases.
\end{abstract}

Keywords: Carbapenemens. Enterobacteriaceae. Klebsiella pneumoniae. Microbial sensitive tests. Drug resistance.

\section{INTRODUCTION}

Emergence of antibiotic-resistant bacteria is a major global public health concern, and the Gram-negative bacilli of the Enterobacteriaceae family are well-known for exhibiting drug-resistance. Drug-resistant microorganisms cause recurrent infections in patients in hospital units, limiting treatment alternatives and increasing morbidity and mortality rates ${ }^{1,2}$

Since the isolation of Enterobacteriaceae that produce an extended spectrum of $\beta$-lactamases (ESBL) capable of hydrolysing almost all cephalosporins, use of carbapenems (imipenem, meropenem, ertapenem, and doripenem) in treating Enterobacteriaceae infections has become mandatory ${ }^{1}$. These

Corresponding author: Dra. Rosmari Hörner.

e-mail: rosmari.ufsm@gmail.com

Received 16 November 2016

Accepted 6 April 2017 antimicrobials are crucial for preventing and treating infections in high-risk patients such as those undergoing transplantation surgery or any other surgical procedure or admitted in intensive care units (ICU) ${ }^{1}$.

A wide variety of carbapenemase-producing Enterobacteriaceae have been reported worldwide ${ }^{3,4}$. Carbapenem resistance is mediated the by transfer of mobile genetic elements such as plasmids and transposons, which are easily transferred to other bacterial genera and species, i.e., Enterobacter cloacae, Citrobacter freundii, Salmonella spp., Escherichia coli, among others.

The mechanisms via which Enterobacteriaceae resists different classes of antimicrobials vary; for example, the mechanisms may be associated with the decrease or loss of porin in bacterial outer membranes (OMPs) and efflux pumps, mutations in the active site of antimicrobials that decreases their affinity for microbes, and the presence of $\beta$-lactamase-encoding genes ${ }^{3,5-7}$. Among the carbapenemases produced from plasmids, the Ambler class A (Klebsiella pneumoniae carbapenemase -KPC and Guiana-Extended-Spectrum-GES) has been identified 
in clinical isolates ${ }^{8}$. The other types of carbapenemases include Ambler class B or metallo- $\beta$-lactamases (MBL) (Verona imipenemase-VIM, Imipinemase-IPM, and New Delhi metallo$\beta$-lactamase-NDM) and oxacillinases or Ambler class D (Oxa-carbapenemases-OXA-48) $)^{1,3,9-11}$.

From the epidemiological point of view, bacteria that produce KPC carbapenemase are the most worrisome owing to their rapid worldwide dissemination ${ }^{12}$. These bacteria are considered important agents of nosocomial infections because they produce carbapenemase, which is an enzyme that hydrolyzes the $\beta$-lactam ring of not only carbapenem antibiotics, but also those of cephalosporins, penicillin, and monobactams ${ }^{13}$.

Infections caused by carbapenem-resistant Enterobacteriaceae (CREs) increase the morbi-mortality rates of patients, especially those admitted in hospitals or with weakened immune systems, and make therapeutic alternatives scarce ${ }^{14,15}$.

In this study, we used phenotypic methods to screen carbapenem-resistant Enterobacteriaceae (CREs) isolated at a university hospital in South Brazil in a one-year period (July 2014 to July 2015), and evaluated their antimicrobial sensitivity profiles.

\section{METHODS}

\section{Study site}

The study was performed at the Laboratório de Bacteriologia do Departamento de Análises Clínicas e Toxicológicas (LaBac) at Centro de Ciências da Saúde of Universidade Federal de Santa Maria (UFSM), Santa Maria, Rio Grande do Sul. Samples were provided by the Laboratório de Análises Clínicas of the Hospital Universitário de Santa Maria (HUSM), Santa Maria, Rio Grande do Sul.

\section{Samples}

One hundred seventy-eight samples of CREs were isolated between July, 2014 and July, 2015 from several biological materials, including epidemiologic vigilance research comprising patients admitted to a university hospital (HUSM) in the southern region of Brazil. Samples were subsequently sent to $\mathrm{LaBac}$ and subsequently stored in $15 \%$ glycerol at $-80^{\circ} \mathrm{C}$ for further phenotypic tests.

\section{Bacterial identification test}

All cultures were collected and processed per the standard operating procedure (SOP) at the Laboratório de Análises Clínicas of the hospital. Identification tests of the isolated bacteria were performed using the automated system, Vitek ${ }^{\circledR} 2$ (BioMérieux, France).

\section{Sensitivity profile}

Sensitivity profiles of the isolates were assessed through the automated methodology Advanced Expert System (BioMérieux, France), following recommendations of the Clinical and Laboratory Standards Institute ${ }^{16}$. Sensitivity cards were used with the following antimicrobials: ertapenem, meropenem, imipenem, amikacin, gentamicin, norfloxacin, nitrofurantoin, sulfamethoxazole/ trimethoprim, ciprofloxacin, tigecycline, and colistin.

\section{Phenotypic tests with phenylboronic acid, cloxacillin, and ethylenediaminetetraacetic acid}

Samples stored in $15 \%$ glycerol at $-80^{\circ} \mathrm{C}$ were reactivated in plates containing trypticase soy agar (TSA/Oxoid LTD, England), and incubated at $35 \pm 2{ }^{\circ} \mathrm{C}$ for 18 to $24 \mathrm{~h}$. A bacterial suspension was subsequently prepared in $0.9 \%$ sterile saline solution, with turbidity similar to the $0.5 \mathrm{McF}$ arland standard, and humidified with a swab sowed in Mueller-Hinton agar (MHA/HiMedia Laboratories, India) in $15 \times 150 \mathrm{~mm}$ plates. Next, ertapenem, meropenem, and imipenem disks (Diagnósticos Microbiológicos Especializados, Brazil) were placed on a Petri dish, supplemented with a $10 \mu \mathrm{L}$ solution of AFB (40mg/mL, Sigma-Aldrich), CLOXA $(75 \mathrm{mg} / \mathrm{mL}$, Sigma-Aldrich), or ethylenediaminetetraacetic acid (EDTA) $(0.1 \mathrm{~mol} / \mathrm{L}$, Proquimios Comércio e Indústria Ltda, Brazil), with a drying time of 20 minutes such that they could be applied on the bacterial suspension in MHA at a distance of $3 \mathrm{~cm}$ from one another. Non-supplemented ertapenem, meropenem, and imipenem disks served for comparison with supplemented disks. In a plate there were placed non-supplemented ertapenem, meropenem, and imipenem disks; ertapenem, meropenem and imipenem disks supplemented with AFB; meropenem and imipenem disks supplemented with CLOXA; and meropenem and imipenem disks supplemented with EDTA. The plates were then incubated at $35 \pm 2^{\circ} \mathrm{C}$ for 18 to $24 \mathrm{~h}^{13}$.

Subsequently, the difference of the inhibition zone diameter was compared between non-supplemented disks and those supplemented with AFB, CLO, or EDTA. Isolates with an inhibition zone difference $\geq 5 \mathrm{~mm}$ for ertapenem, meropenem, and imipenem disks supplemented with AFB were considered possible KPC producers. Isolates with a difference $\geq 5 \mathrm{~mm}$ for antimicrobial disks supplemented with AFB and CLOXA were considered possible producers of plasmid-mediated AmpC. Isolates with zone difference $<5 \mathrm{~mm}$ for antimicrobial disks supplemented AFB, CLOXA and EDTA were considered possible producers of another $\beta$-lactamase (ex. OXA-48) or porin loss, and the ones that showed a zone difference $\geq 5 \mathrm{~mm}$ only for disks supplemented with EDTA were considered likely producers of $\mathrm{MBL}^{13}$.

\section{Ethical considerations}

This study was approved by the Ethical Research Committee of the Federal University of Santa Maria under n ${ }^{\circ}$ 0285.0.243.00009.

\section{RESULTS}

Among the $178 \mathrm{CRE}$ samples analyzed, Klebsiella pneumoniae was the most prevalent microorganism (80.3\%; $\mathrm{n}=143)$, followed by Enterobacter cloacae (8.4\%), Serratia marcescens (5.6\%), Enterobacter aerogenes (2.2\%), Klebsiella oxytoca (1.1\%). Escherichia coli, Salmonella spp., Raoultella ornithinolytica, and Morganella morganii accounted for $0.6 \%$ of the total isolated CREs. Most K. pneumoniae isolates were obtained from the rectal swab $(43.4 \% ; n=62)$, which is a part of surveillance culture, followed by urine $(18.9 \% ; n=27)$ and blood $(10.5 \% ; \mathrm{n}=15)$. The largest number of $E$. cloacae 
was isolated from tracheal secretion $(33.3 \% ; n=5)$, and urine $(26.7 \% ; n=4)$ and rectal swabs $(13.3 \% ; n=2)$ as shown in Table 1.

The growth of $56.7 \%(n=101)$ CREs, which were putative producers of KPC, were inhibited by AFB, whereas $3.4 \%$ $(\mathrm{n}=6)$ were inhibited by EDTA and possibly produced MBL (e.g. NDM, IMP, VIM); further, 7.3\% $(n=13)$ were inhibited by both AFB and CLOXA, and were putative producers of plasmid-mediated AmpC; the growth of $32.6 \%(n=58)$ isolates were not inhibited by AFB, CLOXA, and EDTA, and possibly produced yet another type of $\beta$-lactamase, such as OXA-48 or porin loss (Table 2).

Analysis of the resistance profile of the studied isolates showed that 178 samples showed resistance to at least one carbapenem (ertapenem, meropenem, and imipenem). Among $K$. pneumoniae isolates, $97.9 \%(\mathrm{n}=140)$ showed resistance to ertapenem, $98.6 \%(\mathrm{n}=141)$ to meropenem, and $97.1 \%$ $(\mathrm{n}=101)$ to imipenem, whereas E. cloacae and $S$. marcescens showed $86.7 \%$ and $100 \%$ resistance to ertapenem, respectively. Ciprofloxacin-resistant isolates (90\%) were also detected, as shown in Table 3. Most $K$. pneumoniae isolates were sensitive to aminoglycosides such as amikacin (97.2\%), gentamicin (50\%), and colistin (73.5\%). Only E. cloacae showed low sensitivity to gentamicin in $21.4 \%(\mathrm{n}=3)$ samples.

\section{DIscussion}

The prevalence of CREs has increased worldwide, which represents an alarming threat to public health ${ }^{15}$. In this study, we showed that a large incidence of $K$. pneumoniae was detected in the analyzed samples, and the rectal swab, a surveillance culture, was the clinical material with the maximum number of isolates (43.4\%). The most frequent carbapenemase detected in rectal swab isolates was KPC (64.5\%). Similar results were reported by Pinto et $\mathrm{al}^{15}$, who assessed $701 \mathrm{CREs}$ isolated from hospitals in Porto Alegre, in which $47 \%$ cases were represented by $K$. pneumonia, and $66 \%$ of these were KPC producers. In addition, $51.7 \%$ samples with CREs were from rectal swabs, which corroborated the results of our study ${ }^{15}$. Singh et al. $(2015)^{12}$ evaluated 73 samples from various clinical specimens like urine, pus, swabs, body fluids, among others, in India, of

TABLE 1

Distribution of 178 CREs* isolated at the Hospital Universitário de Santa Maria (HUSM) from July 2014 to July 2015.

\begin{tabular}{|c|c|c|c|c|c|c|c|c|c|}
\hline \multirow[b]{2}{*}{ Clinical supplies } & \multicolumn{7}{|c|}{ Microorganisms } & \multirow[b]{2}{*}{ Salmonella spp } & \multirow[b]{2}{*}{ E. coli } \\
\hline & K. pneumoniae & E. cloacae & S. marcescens & E. aerogenes & K. oxytoce & M. morganii & R.ornithinolytica & & \\
\hline $\begin{array}{l}\text { Swab rectal } \\
\text { (Surveillance } \\
\text { culture) }\end{array}$ & $\begin{array}{c}43.4 \% \\
(n=62)\end{array}$ & $\begin{array}{l}13.3 \% \\
(\mathrm{n}=2)\end{array}$ & $\begin{array}{c}10 \% \\
(n=1)\end{array}$ & $\begin{array}{c}75 \% \\
(n=3)\end{array}$ & - & - & $\begin{array}{l}100 \% \\
(\mathrm{n}=1)\end{array}$ & $\begin{array}{l}100 \% \\
(\mathrm{n}=1)\end{array}$ & - \\
\hline Urine & $\begin{array}{c}18.9 \% \\
(\mathrm{n}=27)\end{array}$ & $\begin{array}{l}26.7 \% \\
(n=4)\end{array}$ & $\begin{array}{c}20 \% \\
(\mathrm{n}=2)\end{array}$ & $\begin{array}{c}25 \% \\
(\mathrm{n}=1)\end{array}$ & - & $\begin{array}{l}100 \% \\
(\mathrm{n}=1)\end{array}$ & - & - & - \\
\hline Blood & $\begin{array}{c}10,5 \% \\
(n=15)\end{array}$ & $\begin{array}{c}6,7 \% \\
(\mathrm{n}=1)\end{array}$ & $\begin{array}{c}40 \% \\
(n=4)\end{array}$ & - & - & - & - & - & $\begin{array}{l}100 \% \\
(\mathrm{n}=1)\end{array}$ \\
\hline $\begin{array}{l}\text { Tracheal } \\
\text { secretion }\end{array}$ & $\begin{array}{c}7.7 \% \\
(\mathrm{n}=11)\end{array}$ & $\begin{array}{l}33.3 \% \\
(\mathrm{n}=5)\end{array}$ & $\begin{array}{c}20 \% \\
(\mathrm{n}=2)\end{array}$ & - & $\begin{array}{c}50 \% \\
(\mathrm{n}=1)\end{array}$ & - & - & - & - \\
\hline Catheter tip & $\begin{array}{c}2.1 \% \\
(\mathrm{n}=3)\end{array}$ & - & - & - & - & - & - & - & - \\
\hline Peritoneal fluid & $\begin{array}{c}1.4 \% \\
(\mathrm{n}=2)\end{array}$ & - & - & - & - & - & - & - & - \\
\hline Secretion wound & $\begin{array}{c}0.7 \% \\
(\mathrm{n}=1)\end{array}$ & $\begin{array}{c}6.7 \% \\
(\mathrm{n}=1)\end{array}$ & - & - & - & - & - & - & - \\
\hline Others* & $\begin{array}{c}7.7 \\
(n=11)\end{array}$ & - & - & - & - & - & - & - & - \\
\hline Total & 143 & 15 & 10 & 4 & 2 & 1 & 1 & 1 & 1 \\
\hline
\end{tabular}

*Carbapenem-resistant Enterobacteriaceae: K. pneumoniae: Klebsiella pneumoniae; E. cloacae: Enterobacter cloacae; $\boldsymbol{S}$. marcescens: Serratia marcescens; E. aerogenes: Enterobacter aerogenes; K. oxytoca: Klebsiella oxytoca; M. morganii: Morganella morganii; R. ornithinolytica: Raoultella ornithinolytica; E. coli: Escherichia coli; -: not done *Muscle tissue; ear secretion; bone tissue; secretion penrose; intraperitoneal secretion; subcutaneous secretion; abdominal aponeurosis; intra-abdominal abscess; swab calcaneus; ascites; peri-prosthetic secretion. 
TABLE 2

Distribution of CREs in clinical specimens and the resistance mechanism obtained in phenotypic tests.

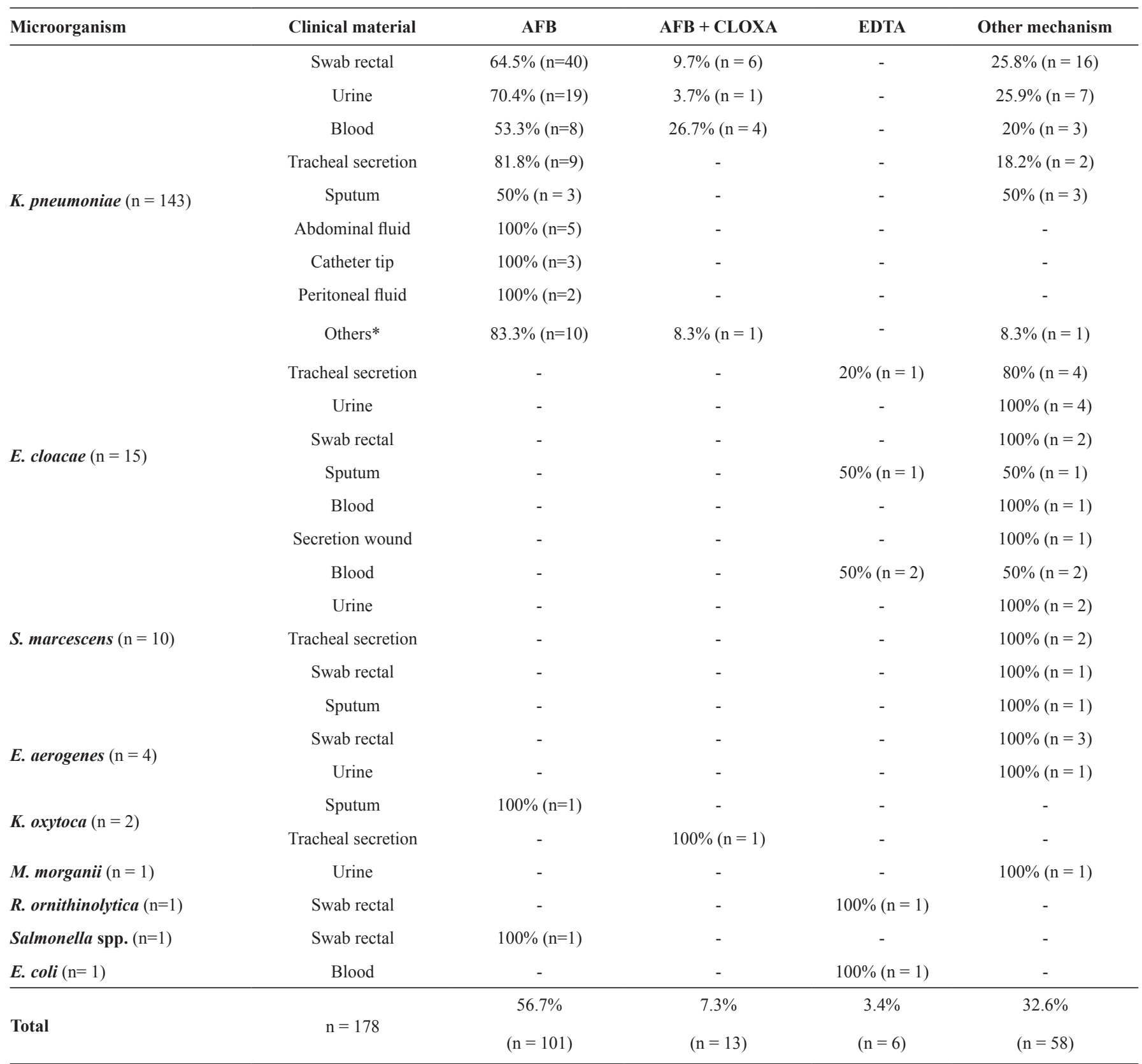

K. pneumoniae: Klebsiella pneumoniae; E. cloacae: Enterobacter cloacae; S. marcescens: Serratia marcescens; E .aerogenes: Enterobacter aerogenes; K. oxytoca: Klebsiella oxytoca; M. morganii: Morganella morganii; R. ornithinolytica: Raoultella ornithinolytica; E. coli: Escherichia coli; AFB: phenylboronic acid; KPC: Klebsiella pneumoniae carbapenemase ; AFB + CLO: phenylboronic acid+ cloxacillin; EDTA: ethylenediaminetetraacetic acid; MBL: metallo$\beta$-lactamases; OXA: oxacillinases. *Secretion of wound; muscle tissue; ear secretion; bone tissue; secretion penrose; intraperitoneal secretion; subcutaneous secretion; abdominal aponeurosis; Intra-abdominal abscess; swab calcaneus; ascites; peri-prosthetic secretion; AFB: possible KPC-producing; AFB + $\mathrm{CLO}=$ possible plasmidial AmpC-producing; EDTA = possible MBL-producing; Other mechanism = production of other $\beta$-lactamase (e.g., OXA-48) or porin loss.

which $41.1 \%(\mathrm{n}=30)$ were KPC-producing K. pneumoniae (via the AFB test), which is similar to the results reported in this study where $56.7 \%$ clinical isolates were found to be KPCpositive using the same test.

Among the isolated CREs, 58 (32.6\%) were carbapenemresistant but were not positive in any phenotypic tests, indicating the presence of another type of $\beta$-lactamases as a resistance mechanism (e.g. OXA-48 or porin loss). This was the secondmost frequent resistance mechanism identified in our study. Since the global frequency of occurrence of this class of carbapenem-resistant bacteria is still low (which corroborates the results of Pinto et al. ${ }^{15}$ ), a detailed investigation into alternative mechanisms of resistance is required to control the dissemination of such strains in future. 
The majority of the isolates showed decreased sensitivity to carbapenems, which are the most commonly used therapeutic choices against these infections ${ }^{1}$. The isolates identified in this study showed increased resistance to carbapenems, quinolones, and glycylcyclines, which is similar to that shown by Hayder et al. ${ }^{17}$, where isolates producing KPC showed $100 \%$ resistance to carbapenems, cephalosporins, quinolones, and penicillin. Our results also in agreement with those reported by Singh et al. ${ }^{12}$, where the greatest resistance was observed for third generation cephalosporins (100\%) and penicillin (93.3\%). In addition, Singh et al. ${ }^{12}$ have verified increased sensitivity to tigecycline $(86.7 \%)$ and polymyxin $(93.3 \%)$, which is different from the results of our study as we observed greater sensitivity to aminoglycosides and colistin.

However, 32 strains isolated in this study were resistant to colistin, which is an antimicrobial used in the treatment of infections caused by multidrug-resistant bacteria. $\mathrm{Liu}^{18}$, observed that the resistance to colistin is mediated by plasmids in K. pneumoniae and E. coli in China, and colistin-resistant bacteria were found in animals and isolated from humans. It is important to highlight that polymyxin B or colistin are used for the treatment of infections caused by CREs and are associated with one or more antimicrobials such as aminoglycosides (gentamicin or amikacin), carbapenems, and tigecycline ${ }^{19,20}$. This increases the concern associated with the indiscriminate use of these drugs in treating nosocomial infections and in veterinary medicine ${ }^{18}$.

The phenotypic detection of CREs is of great importance for clinical laboratories and for monitoring the emergence of resistant bacterial strains. The fast dissemination of genes and mechanisms of resistance to antimicrobials limits therapeutic options and increases the morbi-mortality of patients ${ }^{15}$. Therefore, phenotypic tests that use inhibitors and enhancers of carbapenemases such as AFB, CLOXA, and EDTA are necessary since they provide a good screening method for detection of carbapenemases. In addition, these methods are easy to adapt in the laboratory routine. However, the results obtained from these phenotypic tests should be confirmed by further molecular tests, if required, for identifying resistant strains.

\section{Limitations of the study}

This research presented limitations in terms of non-detection of other resistance mechanisms (OXA, MBL), which could interfere with sensitivity and specificity. In addition, the molecular tests were not performed to confirm the presence of the enzymes.

\section{Acknowledgements}

We gratefully acknowledge the pharmaceutical staff of the Clinical Analyses Laboratory of Hospital Universitário de Santa Maria (HUSM).

\section{Conflict of interest}

The authors declare that there is no conflict of interest.

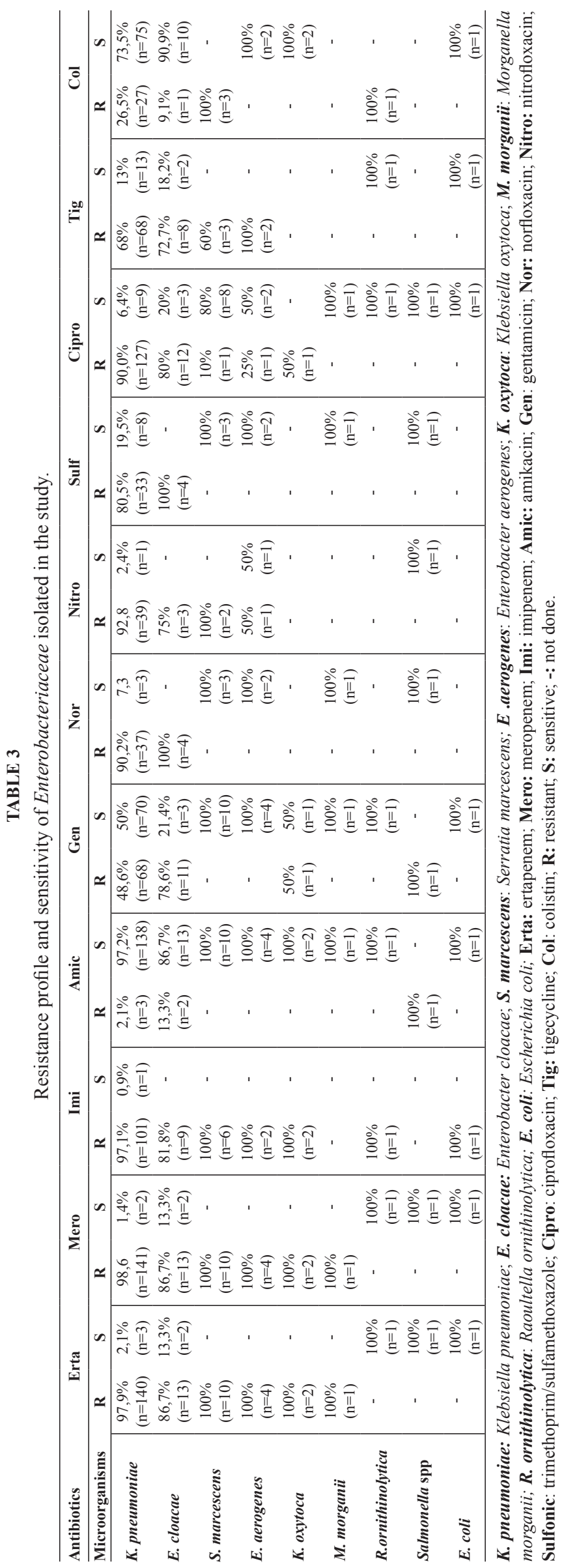




\section{REFERENCES}

1. Nordmann P, Naas T, Poirel L. Global spread of carbapenemase producing Enterobacteriaceae. E Inf Dis. 2011;17(10):1791-98.

2. Birgy A, Bidet P, Genel N, Doit C, Decré D, Arlet G, et al. Phenotypic screening of carbapenemases and associated $\beta$-lactamases in carbapenem-resistant Enterobacteriaceae. J Clin Microbiol. 2012;50(4):1295-1302.

3. Pitout JDD, Nordmann P, Poirel L. Carbapenemase-producing Klebsiella pneumoniae: a key pathogen set for global nosocomial dominance. Antim Ag Chem. 2015;59:5873-84.

4. Guilarde AO, Turchi MD, Martelli CMT, Primo MGB, Batista LJA. Bacteremias em pacientes internados em hospital universitário. Rev Assoc Med Bras. 2007;53(1):34-8.

5. Dienstmann R, Picoli SU, Meyer G, Schenkel T, Steyer J. Avaliação fenotípica da enzima Klebsiella pneumoniae carbapenemase (KPC) em Enterobacteriaceae de ambiente hospitalar. J Bras Patol Med Lab. 2010;46(1):23-7.

6. Ribeiro VB, Zavascki AP, Nodari CS, Sandri AM, Silva MP, Campos JC, et al. Detection of $\mathrm{bla}_{\mathrm{KPC}-2}$ in a carbapenem-resistant Kluyvera georgiana. J Antim Chem. 2012;67(11):2776-7.

7. Tenover FC. Mechanisms of antimicrobial resistance in bacteria. Am J Med. 2006;3(10):62-70

8. Singh M, Kakati B, Agarwal RK, Kotwal A. Detection of Klebsiella pneumoniae carbapenemases (KPCs) among ESBL/MBL producing clinical isolates of Klebsiella pneumoniae. Int J Curr Microbiol App Sci. 2015;4(4):726-31.

9. Clinical and Laboratory Standards Institute (CLSI). Performance Standards for Antimicrobial Susceptibility Testing; Twenty-Fourth Informational Supplement, Document M100-S24. Wayne: CLSI; 2014.

10. Bush K, Jacoby GA. Updated functional classification of $\beta$-lactamases. Antim Agents and Chemother. 2010;54(3):969-76.
11. Hayder N, Hasan Z, Afrin S, Noor R. Determination of the frequency of carbapenemase producing Klebsiella pneumoniae isolates in Dhaka city, Bangladesh. Stamford. J Microbiol. 2012;2(1):28-30.

12. Nordmann P, Poirel L, Dortet L. Rapid detection of carbapenemaseproducing Enterobacteriaceae. E Inf Dis. 2012;18(9):1503-7.

13. Shah AA, Hasan F, Ahmed S, Hameed A. Characteristics, epidemiology and clinical importance of emerging strains of Gramnegative bacilli producing extended-spectrum beta-lactamases. Res Microbiol. 2004;155(6):409-21.

14. Papadimitriou-Olivgeris M, Christofidou M, Fligou F, Bartzavali C, Vrettos T, Filos KS, et al. The role of colonization pressure in the dissemination of colistin or tigecycline resistant KPCproducing Klebsiella pneumoniae in critically ill patients. Infect. 2014;42(7):883-90.

15. Tzouvelekis LS, Markogiannakis A, Piperaki E, Souli M, Daikos GL. Treating infections caused by carbapenemase-producing Enterobacteriaceae. Clin Microbiol Inf. 2014;20(9):862-72.

16. Drawz SM, Bonomo RA. Three decades of beta-lactamase inhibitors. Clin Microbiol Rev. 2010;23(1):160-201.

17. Bartolini A, Frasson I, Cavallaro A, Richter SN, Palù G. Comparison of phenotypic methods for the detection of carbapenem non-susceptible Enterobacteriaceae. Gut Path. 2014;6(13):1-7.

18. Pinto FM, Simas DM, Baldin CP, Limberger II, Silva RCF, Antochevis LC, et al. Prevalence of carbapenemases in carbapenemresistant Enterobacteriaceae in four tertiary care hospitals in Porto Alegre. Clin Biomed Res. 2014;34(1):47-52.

19. Agência Nacional de Vigilância Sanitária (ANVISA). Nota Técnica 01/2013. Medidas de prevenção e controle de infecções por Enterobactérias multiresistentes. Brasília: ANVISA; 2013.

20. Liu YY, Wang Y, Walsh TR, Yi LX, Zhang R, Spencer J, et al. Emergence of plasmid-mediated colistin resistance mechanism MCR-1 in animals and human beings in China: a microbiological and molecular biological study. Lancet Inf Dis. 2016;16(2):161-8. 